\title{
MYBL2 haploinsufficiency increases susceptibility to age-related haematopoietic neoplasia
}

\author{
M Clarke ${ }^{1}$, S Dumon ${ }^{1}$, C Ward ${ }^{1}$, R Jäger ${ }^{2}$, S Freeman ${ }^{1}$, B Dawood ${ }^{1}$, L Sheriff ${ }^{1}$, M Lorvellec ${ }^{1}$, R Kralovics ${ }^{2}$, J Frampton ${ }^{1,3}$ and P García $^{1,3}$
}

The haematopoietic system is prone to age-related disorders ranging from deficits in functional blood cells to the development of neoplastic states. Such neoplasms often involve recurrent cytogenetic abnormalities, among which a deletion in the long arm of chromosome 20 (del20q) is common in myeloid malignancies. The del20q minimum deleted region contains nine genes, including MYBL2, which encodes a key protein involved in the maintenance of genome integrity. Here, we show that mice expressing half the normal levels of $\mathrm{Mybl2}\left(\mathrm{Mybl2}{ }^{+/ \Delta}\right)$ develop a variety of myeloid disorders upon ageing. These include myeloproliferative neoplasms, myelodysplasia (MDS) and myeloid leukaemia, mirroring the human conditions associated with del20q. Moreover, analysis of gene expression profiles from patients with MDS demonstrated reduced levels of MYBL2, regardless of del20q status and demonstrated a strong correlation between low levels of MYBL2 RNA and reduced expression of a subset of genes related to DNA replication and checkpoint control pathways. Paralleling the human data, we found that these pathways are also disturbed in our Myb/2 ${ }^{+/ \Delta}$ mice. This novel mouse model, therefore, represents a valuable tool for studying the initiation and progression of haematological malignancies during ageing, and may provide a platform for preclinical testing of therapeutic approaches.

Leukemia (2013) 27, 661-670; doi:10.1038/leu.2012.241

Keywords: B-Myb; MYBL2; ageing; myelodysplasia; myeloproliferative neoplasm; del20q

\section{INTRODUCTION}

Many blood disorders are associated with ageing. An example of this is myeloid malignancy, where more than $60 \%$ of patients diagnosed with myelodysplastic syndrome (MDS), myeloproliferative neoplasms (MPN) and acute myeloid leukaemia are over 60 years old. ${ }^{1}$ It has been proposed that DNA mutations accumulate during the life of an individual as a consequence of failure to correct errors introduced in the genome during the cell replicative process, leading to either activation of oncogenes or silencing of tumour suppressors that operate as driving factors in the initiation of the disease. ${ }^{2,3}$ Indeed, links have been established between age-related MPNs and specific cytogenetic abnormalities, ${ }^{4}$ and older patients with MPN exhibit a higher frequency of chromosomal defects. ${ }^{5}$

Intriguingly, a percentage of patients with MDS, MPN and myeloid leukaemia present cytogenetic abnormalities that include a deletion in the long arm of chromosome 20 (del20q). ${ }^{6,7}$ It has been proposed that one or more genes within this region might be acting as tumour suppressors, the deletion of which could relate to the onset and/or progression of the disease, although the identity of the gene(s) remains unknown. One gene encoded within del20q with a role in cellular proliferation, DNA replication, maintenance of genome stability and senescence is the transcription factor MYBL2 (B-Myb) ${ }^{8-15}$ Complete deletion of MYBL2 is embryonic lethal. ${ }^{16}$ To assess its role in ageing within the haematopoietic system, we have examined the haematopoietic phenotype in Mybl2 haploinsufficient mice $\left(M y b / 2^{+/ \Delta}\right)$ upon ageing. ${ }^{17}$ We demonstrate that, $M y b / 2^{+/ \Delta}$ mice are prone to develop MDS, MPN and lethal myeloid neoplasm, in contrast to animals expressing normal levels of Mybl2. Moreover, the tumourigenic effects of Mybl2 haploinsufficiency on the haematopoietic system were accelerated when haematopoietic stem cells were subjected to the proliferative stress imposed by bone marrow transplantation. Consistent with this result, we find a strong correlation between MYBL2 expression and 23 genes that are related to DNA replication and cell cycle checkpoint regulation in patients with MDS, regardless of their Del 20q status. Finally, we demonstrate that low MYBL2 expression status correlates with a poor prognosis in patients with high grade MDS (refractory anaemia with excess blasts (RAEB)), regardless of the cytogenetic abnormality. Taken together, our data point to MYBL2 levels as critical in haematopoiesis and suggest a causal role for MYBL2 in the development of a range of haematological malignancies. Our faithful mouse model will not only further inform us of myeloid leukaemogenesis but may also provide a model for preclinical testing of therapeutics.

\section{MATERIALS AND METHODS}

Mice and genotyping

All animal experiments were performed under an animal project licence in accordance with UK legislation. Mice were maintained on a C57/BL6 background. Mice were genotyped by PCR analysis.

\section{Myeloid disorders classification}

Three clinicians (pathologist/haematologists) from the Birmingham Queen's Elizabeth Hospital and Royal Shrewsbury Hospital independently reviewed

${ }^{1}$ Institute of Biomedical Research, Immunity and Infection Department, Birmingham University School of Medical and Dental Science, Edgbaston, Birmingham, UK and ${ }^{2}$ Center for Molecular Medicine of the Austrian Academy of Sciences, Vienna, Austria. Correspondence: Dr P García, Institute of Biomedical Research, Immunity and Infection Department, Birmingham University Medical School, Edgbaston, Birmingham B15 2TT, UK.

E-mail: p.garcia@bham.ac.uk

${ }^{3}$ These two authors are co-senior authors.

Received 14 June 2012; revised 20 July 2012; accepted 7 August 2012; accepted article preview online 22 August 2012; advance online publication, 14 September 2012 
the cases and agreed with the Bethesda criteria for classification of blood disorders observed in $M y b / 2^{+/ \Delta}$ mice.

\section{Blood counts}

Adult mice were bled in acid-citrate-dextrose solution (citric acid $6.8 \mathrm{mM}$ trisodium citrate $11.2 \mathrm{mM}$, glucose $24 \mathrm{mM}$ ) and blood counts obtained with an ABX Pentra 60 (ABX Diagnostics, Irvine, CA, USA) automatic blood counter.

\section{Bone marrow, spleen and liver sections}

Histological analysis of paraffin sections of tibias, spleen and liver was performed using standard methods. H\&E and reticulin-stained sections were analysed under bright field microscopy.

Antibodies, flow cytometry and cell sorting

Single cell suspensions of bone marrow were prepared by standard techniques. Red cells were depleted, when required, by selective lysis. Nonspecific binding of antibodies to Fc receptors was prevented by the use of anti-CD16/CD32 Fc-block (BD Pharmingen, Franklin Lakes, NJ, USA). Antibodies used are described in Supplementary Table 1. Antibodies used for lineage cocktail were B220, Ter119, CD5, CD8a, Gr-1 and Mac-1. Stained cells were analysed on a Cyan flow cytometer using Summit software (Dako, Glostrup, Denmark). For cell sorting, red cells were ACK-lysed (AmmoniumChloride-Potasium) and after staining with the required conjugated monoclonal antibodies, samples were filtered through a 70- $\mu \mathrm{m}$ strainer and sorted on a Cytomation XDP MoFlo machine (Beckman Coulter, Brea, CA, USA). Live cells were selected by forward/side scatter gating and doublet discrimination.

\section{Bone marrow transplants (replication stress assays)}

Transplantations to determine whether replication stress decreases the time of disease appearance were performed by injecting $5 \times 10^{5}$ reference wild-type ACK-treated bone marrow cells (B6 × B6:SJL F1, CD45.1/CD45.2) together with $5 \times 10^{5}$ test donor cells from either wild-type or $M y b / 2^{+/ \Delta}$ mice (B6, CD45.2/CD45.2) into the tail vein of host animals (B6:SJL, CD45.1/ CD45.1) that had been lethally irradiated (975 rads). Peripheral blood was analysed every 4 weeks following transplantation using antibodies to distinguish CD45.1 and CD45.2 and a range of lineage-specific markers. After transplantation, the animals were scored for engraftment by immunofluorescent flow cytometry. Host and donor animals were 8-10 weeks old. Five different donors were used.

\section{Quantitative reverse transcriptase-PCR}

Real time PCR was performed as previously described. ${ }^{11}$ Primers are listed in Supplementary Table 2.

\section{Statistical analysis}

When comparing data sets between wild-type and $M y b / 2^{+/ \Delta}$ animals, two-tailed unpaired Student's $t$-test was used and the unequal variance correction was applied. The significance of difference between expected and observed results was assessed by the $\chi^{2}$ test. When comparing more than two groups at the same time, Kruskal-Wallis one-way analysis of variance on ranks was used to calculate the significance of our results. For the Kaplan-Meier survival curve, statistical significance was determined with the log rank test. For all tests, a $P$ value lower than 0.05 was considered significant.

\section{Microarray}

Preprocessed gene expression data for $\mathrm{CD}_{34}{ }^{+}$cells from 183 patients with MDS and 17 healthy controls were obtained from the Gene Expression Omnibus (GEO) Data sets (GSE19429). ${ }^{18}$

\section{Databases}

Pathway enrichment was determined through query to the Analytical Web portal for high-throughput biology (www.bioprofiling.de), using R spider Tool combining signaling and metabolic pathways from Reactome and KEGG databases. ${ }^{19}$

\section{RESULTS}

The MYBL2 gene is expressed in human and mouse bone marrow progenitor cells

The deletion of the long arm of chromosome 20 (del20q) is variable, but all patients contain a common deleted region (CDR). To date, five CDRs have been described in relation to del20q, each of them containing the MYBL2 gene (Supplementary Figure 1a). 5,7,20-22 To investigate whether any of these genes is expressed in haematopoietic cells, we measured the expression of all nine genes contained within the smallest CDR published so $\mathrm{far}^{7}$ (PTPRT, SFRS6, L3MBTL, SGK2, IFT52, MYBL2, GTSF1L, TOX2 and $\mathrm{JPH} 2$ ), in human $\mathrm{CD}_{3} 4^{+}$and $\mathrm{CD}_{3} 4^{-}$cells and granulocytes, as well as in mouse lineage-negative progenitor cells and more committed lineage-positive cells. Only five of these nine genes, including MYBL2, are expressed in human $\mathrm{CD}_{3}{ }^{+}$cells (Supplementary Figure 1b). Similarly, mouse bone marrow lineage-negative progenitor cells show expression of the same five genes (Supplementary Figure 1c).

Ageing $M y b / 2^{+/ \Delta}$ mice develop a mixed haematological malignant phenotype

As MYBL2 is present in haematopoietic cells and the gene is contained within the CDR of del20q, we decided to use our genetic modification of the MYBL2 locus to determine the importance of Myb/2 haploinsufficiency in the development of myeloid malignancy during ageing. Mybl2 $2^{+/ \Delta}$ mice were first generated by crossing $M y b / 2^{+/ F}$ mice ${ }^{17}$ with $Z$ p3Cre transgenic animals to bring about deletion of the Myb/2 floxed allele at the zygote stage, ${ }^{23}$ whereby germline transmission was achieved, generating mice heterozygous for the deleted allele. As shown in Supplementary Figure 2a, Mybl2 haploinsufficiency correlates with an almost $50 \%$ reduction of Mybl2 mRNA levels. However, full blood counts (Supplementary Figure $3 a$ ) and bone marrow progenitor analysis (Supplementary Figure 3b) did not show any statistically significant cellular changes in animals at 3, 6 or 12 months of age, indicating that a reduction of MYBL2 levels does not impair haematopoietic development or immediately lead to myeloid disorders.

To assess the impact of Myb/2 haploinsufficiency during ageing, a cohort of 24 mice $\left(13 \mathrm{Mybl} 2^{+/ \Delta}\right.$ and $11 \mathrm{Mybl} 2^{+/+}$littermate controls) was maintained for up to 22 months. At that time, mice were killed for analysis, although three of the animals were killed 1 month earlier upon presenting signs of distress (Supplementary Figure 4).

Haematological disorders were evident in 12 out of the 13 Mybl2 ${ }^{+/ \Delta}$ mice, compared to only 1 of the controls $\left(\chi^{2}=16.62\right.$, $P$-value 0.000045$)$ with 9 out of $13 \mathrm{Mybl} 2^{+/ \Delta}$ mice having enlarged spleens compared to only 1 of the controls $\left(\chi^{2}=8.86, P\right.$-value $0.0029)$. The average spleen weight in wild-type mice $(0.1 \pm 0.02 \mathrm{~g})$ being in sharp contrast to the mean of $0.24 \pm 0.12 \mathrm{~g}$ in 8 out of 12 of the $M y b / 2^{+/ \Delta}$ animals, and an extreme case with a spleen of $1.3 \mathrm{~g}$. Using the Bethesda classification criteria, ${ }^{24}$ we ascertained that six of the animals developed MDS $(6 / 13,46 \%)$, five had a MPN $(5 / 13,38 \%)$ and one animal developed a myeloid leukaemia (1/13, 7\%) (Supplementary Table 3).

In grouping the animals by type of disease, we could determine that the decrease in WBC and RBC observed in MDS mice were statistically significant, mirroring the common anaemia and leucopenia seen in the human disease. In addition, in the animals with MPN, a statistically significant increase in WBC and a decrease of RBC were observed mirroring haematological indices in some human MPN (Figure 1a). By contrast, platelet numbers varied greatly within each disease-group, with some animals showing thrombocytopenia and others thrombocytosis (Figure 1a). However, this platelet heterogeneity is not uncommon in human MPN and MDS subtypes. By analysing the progression of WBC, RBC and platelet numbers at established time-points during the life of the 
a

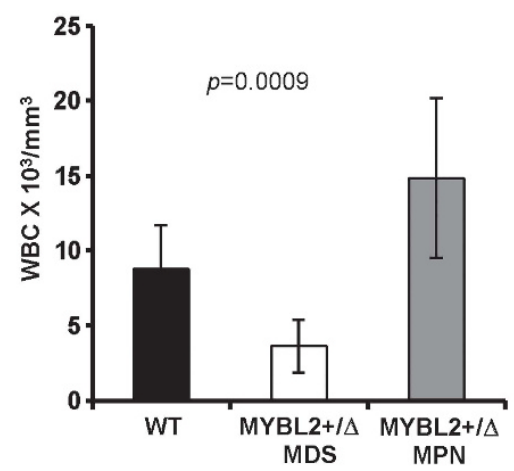

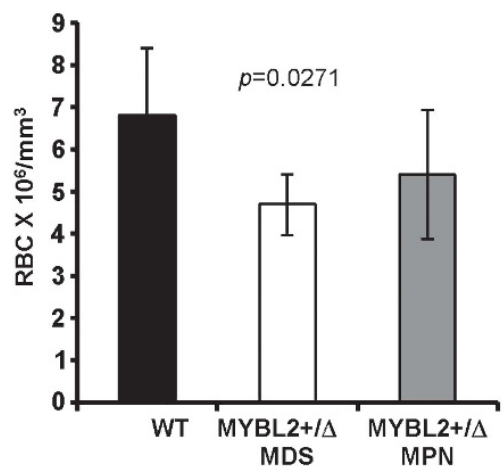
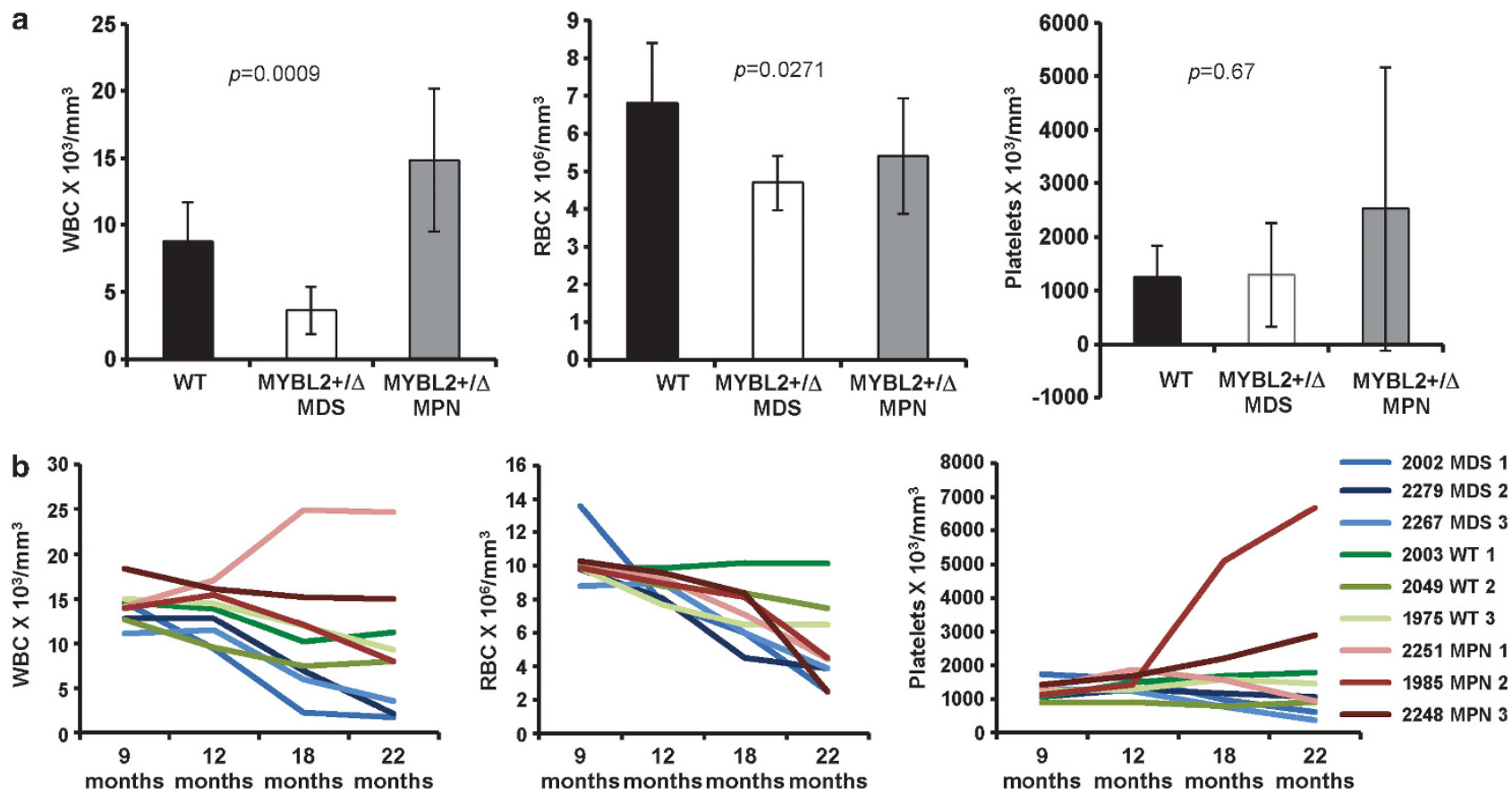

Figure 1. Blood values in ageing mice by disease. (a) Cell counts of WBC (left panel), RBC (middle panel) and platelets (right panel) from the wt (black bars), Mybl2 ${ }^{+/ \Delta}$ mice that developed MDS (white bars) and the $M y b / 2^{+/ \Delta}$ mice that developed MPN (grey bars). Kruskal-Wallis test was used to calculate $P$-value. (b) Charts showing blood values progression of WBC (left panel), RBC (middle panel) and platelets (right panel) at different time-points during the life of three $M y b / 2^{+/ \Delta}$ mice that developed MDS (blue), three Myb/2 ${ }^{+/ \Delta}$ mice that developed MPN (red) and three wild-type mice (green). Each colour represents one mouse.

animals, we could determine that signs of anaemia and changes in WBC counts were detectable between 12 and 18 months of age and exacerbated by 22 months (Figure 1b). We also observed that, regardless of the type of disease developed, no re-expression of Mybl2 or compensation from the other allele took place (Supplementary Figure 2b). It was possible that the loss of sequences within the Mybl2 locus in our mouse model removed a regulatory element important for the expression of neighbouring genes. If that would be the case, the affected gene might effectively participate with Mybl2 in the development of the disease. To rule out this possibility, we measured mRNA expression of these genes in diseased mice. Out of four potentially expressed neighbouring genes, expression of $\mathrm{L3mbtl}$ and Gtsfil could not be detected in wild-type mice, Srsf6 and Itf52 were expressed, but the level of mRNA was not altered in the ageing mice with either the MPN or MDS phenotypes when compared to equivalent age wild-type littermate animals (Supplementary Figure 5), confirming that the only gene deregulated in diseased mice was Mybl2.

Haploinsufficiency of Myb/2 (Myb/2 $\left.{ }^{+/ \Delta}\right)$ predisposes for multiple myeloid disorders

As described above, during ageing, $M y b / 2^{+/ \Delta}$ mice developed MDS, MPN and myeloid neoplasms. The haematological characteristics of mice within each disease-group are summarised below.

MDS in aged $M y b / 2^{+/ \Delta}$ mice

Six out of thirteen $M y b / 2^{+/ \Delta}$ mice developed MDS, mirroring the haematological characteristics and heterogeneity of human MDS disease. The peripheral blood of these animals exhibited neutropenia, and displayed dyserythropoiesis that included size variation (anysocytosis), shape variation (poikilocytosis) and the presence of Howell-Jolly bodies (Figure 2a). Platelet counts were also affected, with animals showing either thrombocytopenia $\left(390 \times 10^{3} / \mathrm{mm}^{3}\right.$ platelets) or thrombocytosis $\left(2900 \times 10^{3} / \mathrm{mm}^{3}\right.$ platelets).

Flow cytometric analyses of peripheral blood and spleen revealed an increase in myeloid populations in $M y b / 2^{+/ \Delta}$ mice compared with controls. The affected myeloid population from diseased mice differed in its surface marker signature, varying between $\mathrm{Mac}^{+}{ }^{+} \mathrm{Gr} 1^{-}$, Mac1 ${ }^{-} \mathrm{Gr}_{1}{ }^{+}$or $\mathrm{Mac}^{+}{ }^{+} \mathrm{Gr} 1^{+}$(Figures $2 \mathrm{~b}$ and c). Further, one animal displayed a striking case of dyserythropoiesis in the spleen, with an abnormal erythroid maturation profile made up of cells expressing lower levels of both Ter119 and CD71 (red circle) (Figure 2d). To determine the aggressiveness of MDS in these animals, we performed bone marrow transplants into sublethally irradiated hosts. The transplanted cells engrafted poorly, even from animals with a more severe phenotype, and did not show any proliferative advantage 8 weeks after transplantation (Supplementary Figure 7a), mimicking the inability of human MDS cells to induce clinical disease in xenotransplants.

MPN in aged Mybl2 $2^{+/ \Delta}$ animals

Five out of thirteen $M y b / 2^{+/ \Delta}$ animals developed a MPN. H\&E staining of bone marrow sections revealed that while aged wildtype mice displayed accumulation of fat cells, Mybl2 $2^{+/ \Delta}$ animals exhibited myeloproliferation with high cellularity and practically no fat cells (Figure 3a). Reticulin staining of bone marrow sections was negative, indicating lack of fibrosis (Supplementary Figure 6b). The heterogeneity observed in MPN patients was also observed in MPN Mybl2 ${ }^{+/ \Delta}$ mice. Thus, three mice had 
a
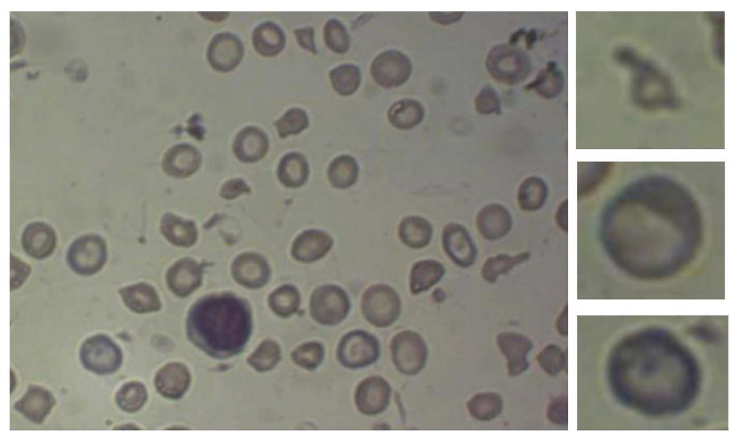

b
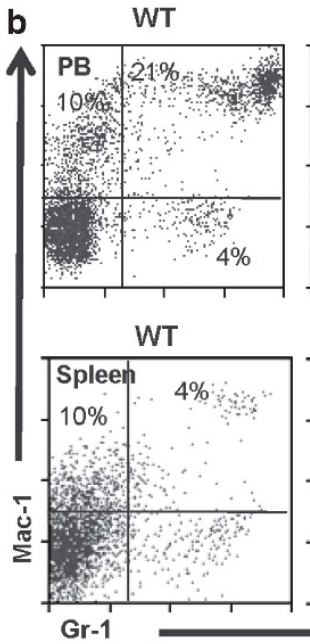

MYBL2 +/D

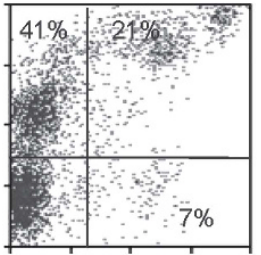

MYBL2 +/ $\triangle$

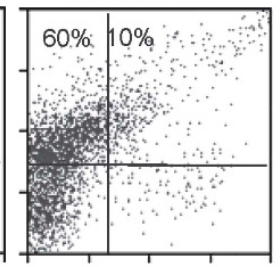

MYBL2 +/A

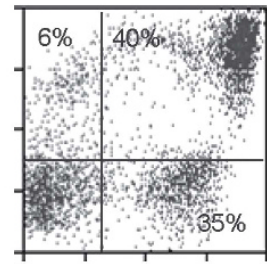

MYBL2 $+/ \Delta$

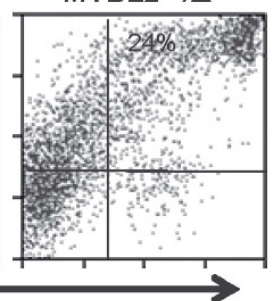

c
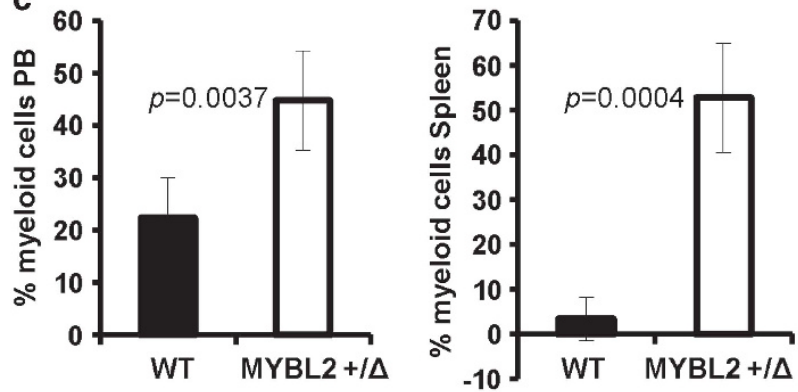

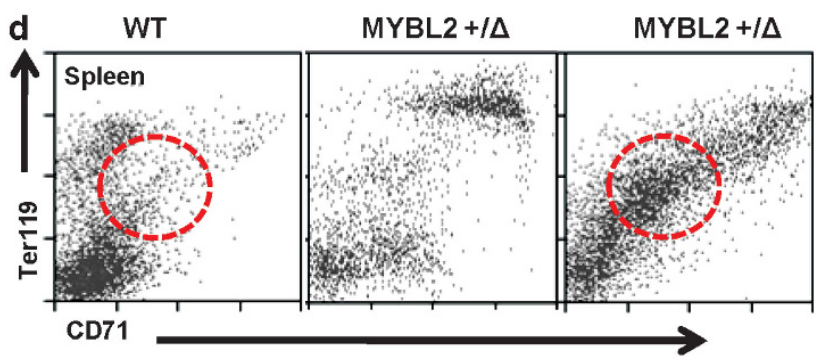

Figure 2. Ageing $M y b / 2^{+/ \Delta}$ mice are prone to develop MDS. (a) Peripheral blood smear from a Mybl2 ${ }^{+/ \Delta}$ mouse stained with DiffQuick where anysocytosis and poikilocytosis are present. (b) Characterisation using Gr-1 and Mac-1 surface markers of peripheral blood and spleen from wild-type and two different $M y b / 2^{+} / \Delta$ animals. (c) Charts showing the percentage of myeloid cells on peripheral blood and spleen from wildtype and Myb/2 $2^{+/ \Delta}$ animals. (d) Characterisation using CD71 and Ter119 surface markers of spleen erythroid cells from wild-type and two different $M y b / 2^{+/ \Delta}$ animals.

leukocytosis with WBC counts as high as $25 \times 10^{3} / \mathrm{mm}^{3}$, with or without anaemia, with or without thrombocytopenia. Flow cytometric analyses revealed an increase in the myeloid population in peripheral blood, bone marrow and spleen (Figure 3b).

The remaining two MPN mice constituted a separate group displaying severe thrombocytosis resembling human essential thrombocythemia, with platelet values as high as 3000 and $6990 \times 10^{3} / \mathrm{mm}^{3}$ (normal levels in ageing mice being 1000 $1500 \times 10^{3} / \mathrm{mm}^{3}$ ) and a MPV of $13.2 \mu \mathrm{m}^{3}$ (normal values around $\left.6 \mu \mathrm{m}^{3}\right)$. A high content of megakaryocytes was detected in the spleen by H\&E staining of tissue sections (Figure $3 c$ ) and in the bone marrow by flow cytometry (Figure $3 \mathrm{~d}$ ).

Bone marrow transplantation into sublethally irradiated hosts showed a good engraftment (50\%) of the MPN cells at 8 weeks after transplantation, in clear contrast with the poor engraftment of the MDS cells (Supplementary Figure 7b). Nevertheless, no signs of MPN disease were observed. No increase in the percentage of the MPN transplanted population was detected even 8 months after transplantation and the transplanted animals did not die as a consequence of the transplanted cells.

\section{Lethal myeloid neoplasm in one Mybl2 ${ }^{+/ \Delta}$ mouse}

One of the aged $M y b / 2^{+/ \Delta}$ mice developed a myeloid neoplasm with marked leukocytosis, anaemia and an increase in the number of platelets. A mild increase in the number of platelets and the tendency to anaemia could be observed at 12 and 18 months of age, whereas the massive increase of WBC started after 18 months (Supplementary Figure 8a), only 3 months before the animal had to be killed due to signs of distress.

Blood smears showed the presence of immature myeloid cells (Supplementary Figure $8 \mathrm{~b}$ ). The profound anaemia in this Mybl2 ${ }^{+/ \Delta}$ mouse was reflected by the white appearance of the bones (Supplementary Figure $8 \mathrm{c}$ ). This mouse also developed splenomegaly (Supplementary Figure 8d), and H\&E staining showed a disruption of spleen architecture, with loss of lymphocytic follicles and an increase in the number of megakaryocytes (Supplementary Figure 8e). Likewise, staining of liver sections showed infiltration of myeloid cells (Supplementary Figure 8f).

The overall profile of bone marrow represented by the side versus forward scatter plots revealed that the $M y b / 2^{+/ \Delta}$ cells were homogeneous, a feature typical of myeloid leukaemia (Supplementary Figure 8g). Furthermore, we detected a dramatic increase in the percentage of monocytes and granulocytes, with $\mathrm{Gr}^{+}{ }^{+} \mathrm{Mac}^{+}$cells constituting more than $50 \%$ of the total population in peripheral blood, bone marrow, spleen and liver (Supplementary Figure 8h), which is in agreement with the observations from blood smears and sections. Together with the high platelet numbers and increased spleen megakaryocytes, these features were collectively suggestive of a MPN that had progressed to a myeloid leukaemia, a progression that also occurs in human MPN patients. 

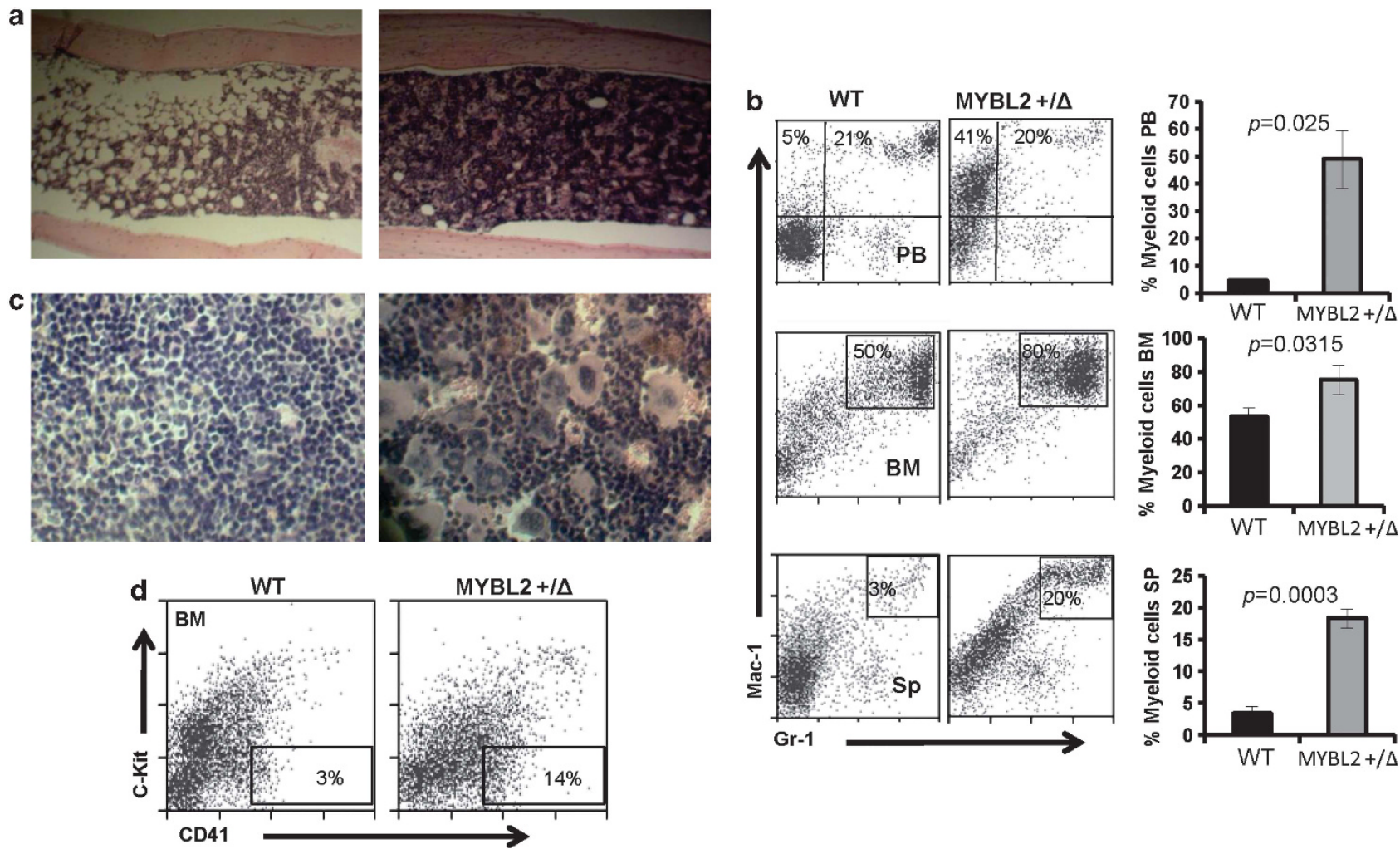

Figure 3. Ageing Mybl2 $2^{+/ \Delta}$ mice develop MPN. (a) H\&E staining of bone marrow paraffin sections from wild-type littermate controls (left panels) and $\mathrm{Mybl2}{ }^{+/ \Delta}$ showing myeloproliferation (right panels). (b) Characterisation using Gr1 and Mac-1 surface markers of peripheral blood (PB), bone marrow (BM) and spleen (Sp) from wild-type and Mybl2 ${ }^{+/ \Delta}$ MPN animals. (c) H\&E staining of spleen paraffin sections from wild-type littermate controls (left panels) and $M y b / 2^{+/ \Delta}$ showing myeloproliferation (right panels). (d) Characterisation using CD41 and c-Kit surface markers of BM from wild-type and a Mybl2 ${ }^{+/ \Delta}$ animal showing high number of platelets in peripheral blood.

Transplantation of bone marrow $M y b / 2^{+/ \Delta}$ cells accelerates development of MDS

As MYBL2 has a role in DNA replication and maintenance of genome integrity, we speculated that the haematological disorders observed in $M y b / 2^{+/ \Delta}$ animals could be the consequence of an accumulation of replication errors. We reasoned that if the phenotype originated in haematopoietic stem cell, as is suggested by the abnormalities of multiple myeloid lineages, then by subjecting the haematopoietic system to replicative stress, we might accelerate disease development. We therefore performed transplantation of total bone marrow cells into lethally irradiated recipients. To detect changes in relative proliferation of the testdonor cells $\left(M y b / 2^{+/+}\right.$or Mybl2 $\left.2^{+/ \Delta}\right)$, we injected these as a $1: 1$ mixture with wild-type reference cells $\left(M y b / 2^{+/+}\right)$. Test-donor and wild-type reference cells could be distinguished by their differential expression of CD45 allelic variants, that is, CD45.2 ${ }^{+}$ (test donor) versus CD45.1 $1^{+} / \mathrm{CD} 45.2^{+}$(reference donor). Host mice were $\mathrm{CD} 45.1^{+}$, which allowed discrimination from transplanted cells. In total, 25 transplanted animals were analysed, of which 10 and 15 had received wild-type or Mybl2 ${ }^{+/ \Delta}$ CD45.2 ${ }^{+}$ donor cells, respectively.

Five out of fifteen (33\%) animals that received $M y b / 2^{+/ \Delta}$ donor cells exhibited a relative increase in the ratio of test:reference reconstitution $\left(\chi^{2}=4.16, P\right.$-value $\left.=0.041\right)$ (Figures $4 a$ and $\left.b\right)$. The mice with increased reconstitution developed MDS 6-9 months after transplantation, whereas none of the 10 animals that were transplanted with control cells showed signs of such a change. Recipients exhibiting the proliferative advantage of the $M y b / 2^{+/ \Delta}$ test cells displayed a phenotype like the ageing mice described above, with splenomegaly (Figure 4c), low WBC counts, anaemia (Figure 4d) and thrombocytopenia. Blood smears showed dyserythropoiesis with signs of polychromasia, anysocytosis and poikilocytosis (Figure 4e).

In the transplanted mice that developed MDS, we determined the contribution of test-donor cells to the erythroid and myeloid lineages by gating on the CD45.1 ${ }^{-} / \mathrm{CD} 45.2^{+}$population. Erythroid lineage maturation appeared to be the most affected, with accumulation of erythroblasts $\left(\mathrm{CD}_{7}{ }^{+} \mathrm{Ter}_{119^{-}}\right)$in the bone marrow, erythropoiesis detected in the spleen and marked dyserythropoiesis (Figure 4f). Unlike reference cells, which yielded lineage cells at the expected turnover, $M y b / 2^{+/ \Delta}$ cells contributed to a marked increase in the proportion of myeloid cells in peripheral blood, bone marrow and spleen (Figure 4g). In addition, animals developing MDS upon transplantation with Mybl2 ${ }^{+/ \Delta}$ cells showed an increased percentage of the $\mathrm{Kit}^{+} \mathrm{Sca} 1^{+} \mathrm{Lin}^{-}$ (KSL) (Figure 4h) or $\mathrm{Kit}^{+} \mathrm{Sca}^{-} \mathrm{Lin}^{-}$(Figure 4i) progenitor populations compared with animals transplanted with control cells, skewing the ratio of KSL:multipotential progenitors from the typical $1: 10$ to above $(1: 3)$ or below $(1: 23)$ the normal ratio (Figure $4 \mathrm{j})$, indicating that the proportion of progenitor cells and KSL cells is deregulated in animals that developed MDS upon transplantation with $M y b / 2^{+/ \Delta}$ cells.

Low MYBL2 expression is detected in human MDS patients suffering RAEB

To test the relevance of our findings in the mouse model for human myeloid disorders, we investigated whether low levels of MYBL2 expression were associated with MPN and MDS in humans, regardless of del20q cytogenetic abnormality. For this purpose, we made use of MDS patient gene-profiling arrays previously published by Pellagatti et al. ${ }^{18}$ Based on the 

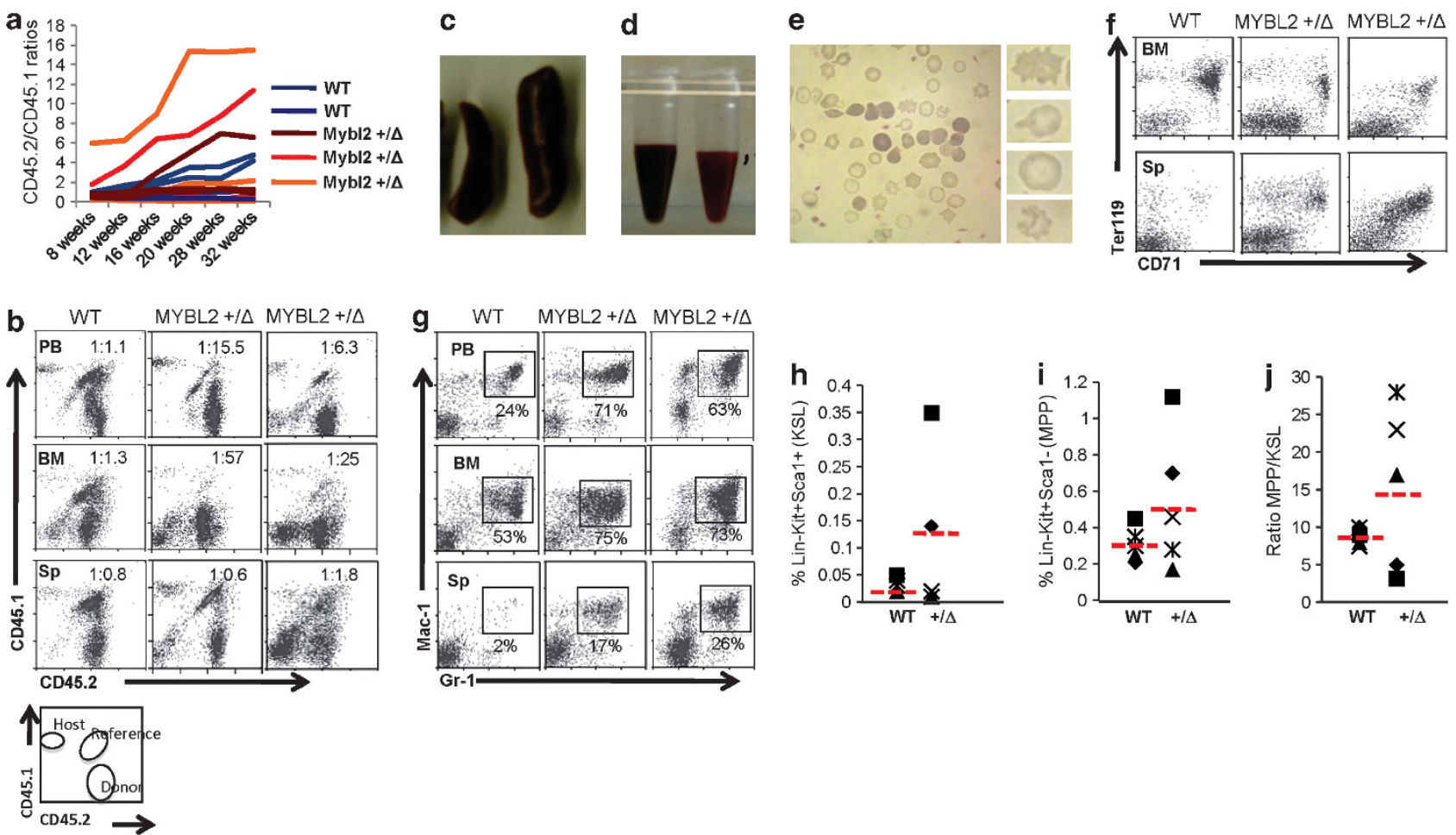

Figure 4. Animals transplanted with $M y b / 2^{+/ \Delta}$ donor cells are more prone to develop MDS disorders. (a) Chart graph shows donor test:reference ratio at different time-points. Two different sets of wild-type donors (blue) and three sets of $M y b / 2^{+/ \Delta}$ donors (red/orange), each carried out in duplicate, are shown. (b) Reference/donor profiles of peripheral blood (PB), bone marrow (BM) and spleen (Sp) at 32 weeks after transplantation from one wild-type donor and two Mybl2 ${ }^{+/ \Delta}$ donors that developed MDS. (c) Picture showing splenomegaly in animals transplanted with $M y b / 2^{+/ \Delta}$ bone marrow cells (right) compared with animals transplanted with wild-type bone marrow cells (left). (d) Pictures showing pale blood from one mouse transplanted with $M y b / 2^{+/ \Delta}$ bone marrow cells (right) compared with normal colour blood from an animal transplanted with wild-type bone marrow cells (left). (e) Diffquick staining of blood film from a mouse transplanted with $M y b / 2^{+} / \Delta$ bone marrow cells showing polychromasia and anysocitosis and poikilocytosis. (f) Analysis of donor (CD45.1 $\left.{ }^{-} / \mathrm{CD}^{2} 5.2^{+}\right)$contribution to the erythroid lineage in BM and Sp in animals transplanted with wild-type donor and two animals transplanted with $M Y B L 2^{+/ \Delta}$ donor cells. (g) Analysis of donor (CD45.1- /CD45.2 $2^{+}$) contribution to myeloid lineages in PB, BM and Sp in animals transplanted with wild-type donor and Mybl2 ${ }^{+/ \Delta}$ donor cells. (h-i) Chart graphs show the percentage of donor (CD45.1 $1^{-} / C D 45.2^{+}$) contribution to (h) KSL cells and (i) multipotential progenitors (MPP) in five animals transplanted with wild-type donor cells and the five animals transplanted with $M y b / 2^{+/ \Delta}$ donor cells that developed MDS. (j) Chart graph shows MPP/KSL ratios. Each symbol refers to individual animals. Red bars represent the average value $(n=5)$.

French-American-British (FAB) cooperative study group classification and its subsequent modifications by the World Health Organisation (WHO), a cohort of 183 MDS patients was subdivided into three categories based on phenotypic characteristics, that is, MDS with refractory anaemia, refractory anaemia with ring sideroblasts and RAEB, the latter being further subclassified into RAEB1 and RAEB2 subgroups according to the percentage of bone marrow blasts (Supplementary Table 4).

Comparison of MYBL2 levels in $\mathrm{CD}_{3}{ }^{+}$cells, from normal donors versus patients within each subgroup, revealed a significant decrease in MYBL2 expression in MDS patients with RAEB, with levels down to 54\% in MDS RAEB2 patients (Figure 5a). In contrast, very little variation was observed in the expression of the other four genes that are associated with del20q in MDS patients (Figure $5 \mathrm{~b}$ ). These findings therefore show a decreased expression of the MYBL2 gene in human MDS subgroups that have been associated with poorer prognosis. ${ }^{25}$

Although MPN data is scarce, by using arrays made public by Guglielmelli et al., ${ }^{26}$ we were able to compare MYBL2 expression in $\mathrm{CD} 4^{+}$cells from three normal donors versus three patients with idiopathic myelofibrosis. Similar to the MDS patients described above, MYBL2 expression appears to be significantly lower in MPN patients (Supplementary Figure 9).
MYBL2 expression correlates with DNA replication and checkpoint proteins in human MDS

In an effort to better understand which biological processes may associate with the low expression level of MYBL2 in MDS, we sought to identify genes whose expression correlated specifically with that of MYBL2 in human MDS. We used the Pearson correlation coefficient as a measure of relationships between levels of expression of different genes, and evaluated the link between expression of each gene against the level of the MYBL2 transcript. A threshold Pearson correlation coefficient of 0.78 (and $P$ value $<0.001)$ was set as a filter to isolate strong and highly significant correlations.

This analysis identified 23 MYBL2-correlated genes in CD34 ${ }^{+}$ cells from patients with MDS RAEB (Figure $6 a$ and Supplementary Figure 10). This list of genes was used to query protein reaction and pathway databases via the $\mathrm{R}$ spider tool, ${ }^{19}$ resulting in the mapping of 16 genes to its reference global network demonstrating a strong enrichment in mitotic $(P=0.01)$, DNA replication $(P=0.01)$ and cell cycle checkpoints $(P=0.015)$ pathways (Figure $6 \mathrm{~b}$ ). Hence, MYBL2 particularly associates with genes regulating the cell cycle of MDS RAEB CD34 ${ }^{+}$cells. To assess whether MYBL2 expression influenced mRNA levels of these genes, we tested their respective expression in MDS cells 
a
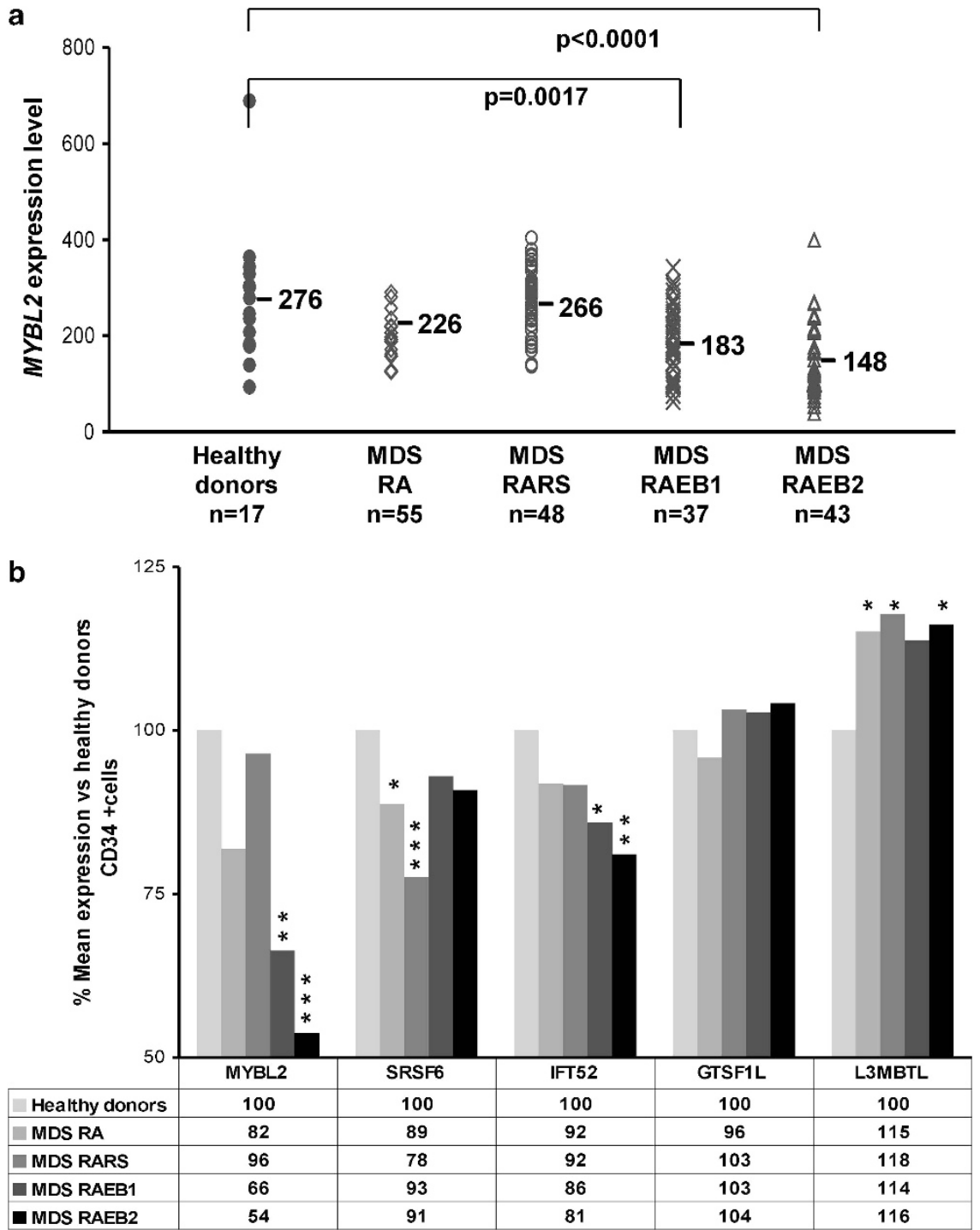

Figure 5. Low MYBL2 expression is detected in human MDS patients with poor prognosis. (a) Chart graph shows MYBL2 expression levels in CD34 + cells from 183 MDS patients, subdivided into different subclassifications according to WHO, compared to CD34 + cells from healthy donors. $P$ values are indicated for statistically significant differences (two-tail Student's $t$-test unpaired unequal variances). (b) Chart graph shows percentage of mean expression of genes contained within del20q minimum common deleted region (MYBL2, SFSR6, ITF52, GTSF1L and L3MBTL) in CD34 + cells from 183 MDS patients, subdivided into different subclassifications according to WHO, compared with CD34 + cells from healthy donors $\left({ }^{*} P<0.05 ;{ }^{*} P<0.01 ;{ }^{* * *} P<0.001\right)$.

from our Mybl2 ${ }^{+/ \Delta}$ ageing mice model. As shown in Figure $6 \mathrm{c}$, the expression of the cell cycle proteins cdc6, Birc 5, cdc20, Ube2C, cycA2 and Orc1L were also downregulated in $M y b / 2^{+/ \Delta}$ cells paralleling the lower expression levels of these genes in human MDS patients.

\section{DISCUSSION}

The work described here shows a direct correlation between MYBL2 expression levels and the occurrence of haematological pathologies such as MPN, MDS and myeloid leukaemia, therefore establishing deregulation of MYBL2 expression as a contributory factor in these myeloid disorders. In humans, these conditions have a higher occurrence in the elderly and a variable tendency of progression to acute myeloid leukaemia in patients diagnosed with MDS or MPN. Strikingly, we observe the same phenotype in mice expressing half the normal levels of Mybl2, providing in vivo evidence that Myb/2 haploinsufficiency triggers haematopoietic defects and induces myeloid malignancies.
The observed phenotype was reminiscent of human disease in more than one aspect. First, transplantation of bone marrow cells from the MDS or MPN mice into sublethally irradiated animals did not result in disease after 8 weeks despite positive engraftment, paralleling the inability of human MDS and MPN cells to induce clinical disease in xenotransplants. ${ }^{27,28}$ Second, we were able to accelerate disease development by inducing replication stress in haematopoietic cells, suggesting that, corresponding to its role in protecting chromosome integrity, ${ }^{10}$ MYBL2 acts as a stabilising factor to prevent accumulation of random secondary mutations that contribute to the development of myeloid malignancies. The random nature of these mutations would also explain the partially penetrant MPN and MDS phenotypes that we have demonstrated. However, the accelerated appearance of hematological disorders only resulted in the appearance of MDS. We speculate that the lack of animals showing MPN disease could be because they required a longer period of time to develop. This could be possible as in our experiments once the first mouse from the cohort showed signs of disease the whole cohort was killed. The MDS 


a
\begin{tabular}{|l|r|r|}
\hline gene_ID & PCC & \multicolumn{1}{l|}{ pval } \\
\hline ESPL1 & 0.79 & $3.01 \mathrm{E}-18$ \\
\hline CDC20 & 0.82 & $2.82 \mathrm{E}-20$ \\
\hline CENPE & 0.82 & $5.77 \mathrm{E}-21$ \\
\hline CDCA3 & 0.78 & $9.43 \mathrm{E}-18$ \\
\hline CDCA5 & 0.83 & $2.04 \mathrm{E}-21$ \\
\hline CDKN3 & 0.79 & $1.63 \mathrm{E}-18$ \\
\hline SPC25 & 0.79 & $3.89 \mathrm{E}-18$ \\
\hline ORC1L & 0.79 & $3.28 \mathrm{E}-18$ \\
\hline CCNA2 & 0.82 & $1.24 \mathrm{E}-20$ \\
\hline CDC6 & 0.81 & $5.58 \mathrm{E}-20$ \\
\hline CDCA8 & 0.83 & $4.91 \mathrm{E}-21$ \\
\hline MKI67 & 0.80 & $5.75 \mathrm{E}-19$ \\
\hline CENPM & 0.82 & $7.81 \mathrm{E}-21$ \\
\hline CCNB2 & 0.78 & $8.86 \mathrm{E}-18$ \\
\hline KIF2C & 0.83 & $3.58 \mathrm{E}-21$ \\
\hline FAM54A & 0.79 & $3.86 \mathrm{E}-18$ \\
\hline MCM10 & 0.79 & $6.73 \mathrm{E}-18$ \\
\hline BIRC5 & 0.80 & $5.87 \mathrm{E}-19$ \\
\hline UBE2C & 0.81 & $1.34 \mathrm{E}-19$ \\
\hline KIF4A & 0.81 & $1.86 \mathrm{E}-19$ \\
\hline CENPF & 0.81 & $9.45 \mathrm{E}-20$ \\
\hline PLK4 & 0.78 & $1.34 \mathrm{E}-17$ \\
\hline NCAPH & 0.83 & $1.10 \mathrm{E}-21$ \\
\hline
\end{tabular}

\begin{tabular}{|c|c|c|c|}
\hline Pathway & $\begin{array}{c}\text { Number } \\
\text { of } \\
\text { genes }\end{array}$ & $\begin{array}{c}\text { gene } \\
\text { names }\end{array}$ & pval \\
\hline $\begin{array}{c}\text { DNA } \\
\text { REPLICATION }\end{array}$ & 12 & $\begin{array}{c}\text { CCNA2 } \\
\text { MCM10 } \\
\text { SPC25 } \\
\text { BIRC5 } \\
\text { CENPF } \\
\text { CDC20 } \\
\text { CDCA8 } \\
\text { CDC6 } \\
\text { CENPE } \\
\text { CENPM } \\
\text { KIF2C } \\
\text { ORC1L }\end{array}$ & 0.01 \\
\hline $\begin{array}{l}\text { CELL CYCLE } \\
\text { MITOTIC }\end{array}$ & 15 & $\begin{array}{l}\text { CCNA2 } \\
\text { MCM10 } \\
\text { SPC25 } \\
\text { BIRC5 } \\
\text { CENPF } \\
\text { CDC20 } \\
\text { PLK4 } \\
\text { CDCA8 } \\
\text { CDC6 } \\
\text { CENPE } \\
\text { CENPM } \\
\text { KIF2C } \\
\text { ORC1L } \\
\text { UBE2C } \\
\text { CCNB2 }\end{array}$ & 0.01 \\
\hline $\begin{array}{l}\text { CELL CYCLE } \\
\text { CHECKPOINTS }\end{array}$ & 6 & $\begin{array}{l}C D C 6 \\
\text { ORC1L } \\
M C M 10 \\
\text { UBE2C } \\
\text { CCNB2 } \\
\text { CDC20 }\end{array}$ & 0.015 \\
\hline
\end{tabular}

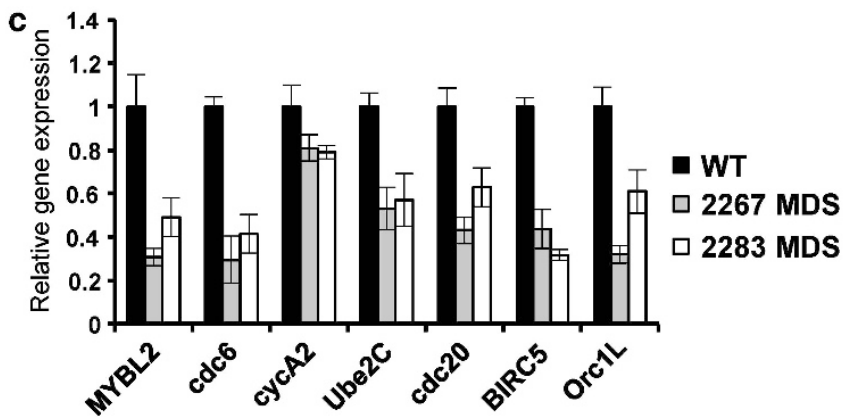

Figure 6. MYBL2 expression signature in MDS. (a) List of genes whose expression correlates highly with MYBL2 expression in MDS, as measured by the Pearson correlation coefficient. (b) Enriched pathways in MYBL2 high correlated genes. (c) Relative gene expression analysis relative to $\beta 2$-microglobulin in Mybl2 ${ }^{+/ \Delta}$ Mac-1 + cells coming from ageing MDS mice. Six cell cycle genes that show high correlation to MYBL2 in human MDS patients were analysed using quantitative PCR.

animals presented an increase in the percentage of the $\mathrm{Kit}^{+} \mathrm{Lin}^{-}$ populations, which is also a feature in patients with MDS. ${ }^{27,28}$

During ageing, there are two major changes in the haemopoietic system: the diminution of the adaptive immune system and an increase in the appearance of myeloproliferative disease. ${ }^{29}$ A simple explanation for the lack of lymphoid malignancies in Mybl2 haploinsufficient mice could be precisely the fact that with age, the haemopoietic system is skewed towards the production of myeloid cells at the expense of the lymphoid lineage. Alternatively, lower levels of Mybl2 may impact differently on specific cell types, having its greatest effect in committed myeloid progenitors.

Importantly, our model of Myb/2 haploinsufficiency recapitulates the appearance of blood disorders in humans during ageing in a way that has distinct advantages over many previously described models. For instance, Pten ${ }^{+/-}$Ship $^{-1-}$ mice $^{30}$ and animals overexpressing Evi1 ${ }^{31}$ have lifespans of only 5 to 11 weeks, preventing their utilisation in long-term studies, as we have described here. Furthermore, blood disorders in these mice cannot be classified as MDS or leukaemia, so that the models fall short of expectations for comparison with human pathologies.
In a recent SNP array-based karyotyping study of MPN, haploinsufficiency has been detected at high frequency for genes encoding transcription factors such as CUX1, IKZF1, ETV6, FOXP1 and RUNX1.5

Del20q is an interstitial deletion commonly found in these disorders, $^{32,33}$ and the minimal CDR has been shown to encompass a number of genes, among which the gene encoding polycomb protein L3MBTL1 is perhaps the best characterised. Interestingly, L3MBTL1 expression appears to be normal in human myeloid malignancies, ${ }^{20}$ which is consistent with our analysis in 183 MDS patients. Moreover, studies using L3mbt/1 knockout mice showed that these do not develop haematological disorders, even when allowed to age for more than 2 years, ${ }^{34}$ indicating that haploinsufficiency of $L 3 \mathrm{mbt} / 1$ does not contribute to the development of haematopoietic disorders in vivo, and that it is not a critical tumour suppressor in the context of del20q in human myeloid malignancies.

Our results demonstrate that (i) the earlier appearance of blood disorders when $M y b / 2^{+/ \Delta}$ cells are subjected to proliferation stress; (ii) lower MYBL2 expression levels in MDS patients with no del20q abnormality; and (iii) significant correlation between 
expression levels of MYBL2 and a subset of DNA replication/cell cycle regulation genes in human MDS patients indicate that MYBL2 indeed acts to protect against haematological malignancy. In MDS human patient samples, a deregulation of cell survival and cell cycle regulation pathways has been reported. ${ }^{18}$ In fact, some of the genes that signature correlates with MYBL2, such as birc5 and cdc20, have been described to be downregulated in MDS patients. ${ }^{35,36}$ This positive correlation is in agreement with a known role for MYBL2 in cell proliferation and genome stability. ${ }^{8-15}$ Although these two genes, birc 5 and cdc20, have not been shown to be direct targets of MYBL2, they are targets of FoxM1, a gene which is a master regulator of the G2/M phase and previously shown to be transcriptionally regulated by MYBL2 in embryonic stem cells. ${ }^{11}$

Just recently, Down et $a .^{37}$ have described that in the absence of MYBL2, FoxM1 cannot bind to nor activate birc5. This study proposes that MYBL2 is required as a pioneer factor to enable FoxM1 binding to G2/M gene promoters. ${ }^{37}$ This could explain the large number of FoxM1 known target genes (Ube2c, cdc20, birc5, cenpF, cyclin B2) that correlated with the MYBL2 signature in MDS. In this way, the control of cell division might be impaired by low levels of MYBL2, altering cell fates established during cell division in early haematopoietic stem and progenitor cells that lead to clonal expansion with imbalance or impaired differentiation.

Our results linking MYBL2 deficiency with development of MPN and MDS are consistent with recent data from the group of Thomas Look (http://ash.confex.com/ash/2010/webprogram/ Paper31436.html), who showed that MYBL2 levels were downregulated in about $70 \%$ of MDS patients. Moreover, two different point mutations in the MYBL2 gene were identified in a subset of patients with MDS who lacked del20q.

MYBL2 has recently been reported to be negatively regulated by two different miRNAs, miR-29 and miR-30. ${ }^{15,38}$ In this context, Slape and colleagues showed up regulation of both miRNAs during leukaemic transformation. It is possible that downregulation of MYBL2 upon overexpression of miR-29 may account for the development of haematological neoplasms in human patients that lack del20q deletions or mutations of MYBL2. This observation has clinical implications, as it illustrates how individual factors may still intervene as a molecular switch in the onset of blood pathologies without prior damage to their primary gene structure, thus rendering the gene undetectable to genomewide sequencing approaches that aim to define the molecular basis of the disease and to find specific gene mutations with diagnostic or prognostic value. Indeed, our observation that MYBL2 levels are downregulated in those MDS patients with poor prognosis (RAEB), regardless of cytogenetic abnormalities, indicates that mechanisms other than gene deletion must operate that bring MYBL2 levels down.

Recent reports have pointed out that mutations in the splicing factor gene SF3B1 are present in 20\% of MDS patients, and with a high frequency $(65 \%)$ in those patients that present ring sideroblasts, which is the subtype with better prognosis. ${ }^{39-41}$ This is in clear contrast with MYBL2 gene, which is significantly downregulated in those patients with a worse prognosis (RAEB). Thus, mutations in SF3B1 might be an indicative of a more favourable prognosis in MDS, whereas downregulation of MYBL2 might suggest a worse prognosis. If validated through clinical observation, both genes could potentially serve as prognostic biomarkers of disease progression and have an impact in prospective therapeutic strategies for MDS patients.

In summary, we have generated mice haploinsufficient for Mybl2 that display age-related haematological neoplasms reminiscent of human disease. MYBL2 seems to be acting as a triggering factor in the onset of blood malignancies through its ability to affect the expression of genes linked to DNA-replication and check-point control, ultimately impairing the physiological balance of blood cell turnover. Our model system could represent a valuable tool for identifying collaborative mutations that define the development of a particular MPN, MDS or transformation to leukaemia.

\section{CONFLICT OF INTEREST}

The authors declare no conflict of interest.

\section{ACKNOWLEDGEMENTS}

We thank Constance Bonifer and Brian Huntly for critical reading of the manuscript, and Oscar Berlanga, freelance medical writer, for editing services. This work was supported by Leukaemia and Lymphoma Research and Wellcome Trust and through a Science City Research Alliance fellowship to PG.

\section{REFERENCES}

1 Rollison DE, Howlader N, Smith MT, Strom SS, Merritt WD, Ries LA et al. Epidemiology of myelodysplastic syndromes and chronic myeloproliferative disorders in the United States, 2001-2004, using data from the NAACCR and SEER programs. Blood 2008; 112: 45-52.

2 Kenyon J, Gerson SL. The role of DNA damage repair in aging of adult stem cells. Nucleic Acids Res 2007; 35: 7557-7565.

3 Maslov AY, Vijg J. Genome instability, cancer and aging. Biochim Biophys Acta. 2009; 1790: 963-969.

4 Dunlap J, Kelemen K, Leeborg N, Braziel R, Olson S, Press R et al. Association of JAK2 mutation status and cytogenetic abnormalities in myeloproliferative neoplasms and myelodysplastic/myeloproliferative neoplasms. Am J Clin Pathol 2011; 135: 709-719.

5 Klampfl T, Harutyunyan A, Berg T, Gisslinger B, Schalling M, Bagienski K et al. Genome integrity of myeloproliferative neoplasms in chronic phase and during disease progression. Blood 2011; 118: 167-176.

6 Aatola M, Armstrong E, Teerenhovi L, Borgstrom GH. Clinical significance of the del(20q) chromosome in hematologic disorders. Cancer Genet Cytogenet 1992; 62: 75-80.

7 Bench AJ, Nacheva EP, Hood TL, Holden JL, French L, Swanton S et al. Chromosome 20 deletions in myeloid malignancies: reduction of the common deleted region, generation of a PAC/BAC contig and identification of candidate genes. UK Cancer Cytogenetics Group (UKCCG). Oncogene 2000; 19: 3902-3913.

8 Lam EW, Bennett JD, Watson RJ. Cell-cycle regulation of human B-myb transcription. Gene 1995; 160: 277-281.

9 Fung SM, Ramsay G, Katzen AL. Mutations in Drosophila myb lead to centrosome amplification and genomic instability. Development 2002; 129: 347-359.

10 Garcia P, Frampton J. The transcription factor B-Myb is essential for S-phase progression and genomic stability in diploid and polyploid megakaryocytes. J Cell Sci 2006; 119: 1483-1493.

11 Lorvellec M, Dumon S, Maya-Mendoza A, Jackson D, Frampton J, Garcia P. B-Myb is critical for proper DNA duplication during an unperturbed $S$ phase in mouse embryonic stem cells. Stem Cells 2010; 28: 1751-1759.

12 Manak JR, Wen H, Van T, Andrejka L, Lipsick JS. Loss of Drosophila Myb interrupts the progression of chromosome condensation. Nat Cell Biol 2007; 9: 581-587.

13 Sala A. B-MYB, a transcription factor implicated in regulating cell cycle, apoptosis and cancer. Eur J Cancer 2005; 41: 2479-2484.

14 Huang Y, Wu J, Li R, Wang P, Han L, Zhang Z et al. B-MYB delays cell aging by repressing p16 (INK4alpha) transcription. Cell Mol Life Sci 2010; 68: 893-901.

15 Martinez I, Cazalla D, Almstead LL, Steitz JA, DiMaio D. miR-29 and miR-30 regulate B-Myb expression during cellular senescence. Proc Natl Acad Sci USA 2011; 108: 522-527.

16 Tanaka Y, Patestos NP, Maekawa T, Ishii S. B-myb is required for inner cell mass formation at an early stage of development. J Biol Chem 1999; 274: 28067-28070.

17 Garcia P, Berlanga O, Watson R, Frampton J. Generation of a conditional allele of the B-myb gene. Genesis 2005; 43: 189-195.

18 Pellagatti A, Cazzola M, Giagounidis A, Perry J, Malcovati L, Della Porta MG et al. Deregulated gene expression pathways in myelodysplastic syndrome hematopoietic stem cells. Leukemia 2010; 24: 756-764.

19 Antonov AV, Schmidt EE, Dietmann S, Krestyaninova M, Hermjakob H. R spider: a network-based analysis of gene lists by combining signaling and metabolic pathways from Reactome and KEGG databases. Nucleic Acids Res 2010; 38: W78-W83.

20 MacGrogan D, Kalakonda N, Alvarez S, Scandura JM, Boccuni P, Johansson B et al. Structural integrity and expression of the L3MBTL gene in normal and malignant hematopoietic cells. Genes Chromosomes Cancer 2004; 41: 203-213.

21 Kilpivaara O, Mukherjee S, Schram AM, Wadleigh M, Mullally A, Ebert BL et al. A germline JAK2 SNP is associated with predisposition to the development 
of JAK2(V617F)-positive myeloproliferative neoplasms. Nat Genet 2009; 41: 455-459.

22 Stegelmann F, Bullinger L, Griesshammer M, Holzmann K, Habdank M, Kuhn S et al. High-resolution single-nucleotide polymorphism array-profiling in myeloproliferative neoplasms identifies novel genomic aberrations. Haematologica 2010; 95: 666-669.

23 Lewandoski M, Wassarman KM, Martin GR. Zp3-cre, a transgenic mouse line for the activation or inactivation of loxp-flanked target genes specifically in the female germ line. Curr Biol 1997; 7: 148-151.

24 Kogan SC, Ward JM, Anver MR, Berman JJ, Brayton C, Cardiff RD et al. Bethesda proposals for classification of nonlymphoid hematopoietic neoplasms in mice. Blood 2002; 100: 238-245.

25 Greenberg P, Cox C, LeBeau MM, Fenaux P, Morel P, Sanz G et al. International scoring system for evaluating prognosis in myelodysplastic syndromes. Blood 1997; 89: 2079-2088.

26 Guglielmelli P, Zini R, Bogani C, Salati S, Pancrazzi A, Bianchi E et al. Molecular profiling of $\mathrm{CD} 34+$ cells in idiopathic myelofibrosis identifies a set of diseaseassociated genes and reveals the clinical significance of Wilms' tumor gene (WT1). Stem Cells 2007; 25: 165-173.

27 Thanopoulou E, Cashman J, Kakagianne T, Eaves A, Zoumbos N, Eaves C. Engraftment of NOD/SCID-beta2 microglobulin null mice with multilineage neoplastic cells from patients with myelodysplastic syndrome. Blood 2004; 103: 4285-4293.

28 Kerbauy DM, Lesnikov V, Torok-Storb B, Bryant E, Deeg HJ. Engraftment of distinct clonal MDS-derived hematopoietic precursors in NOD/SCID-beta2-microglobulindeficient mice after intramedullary transplantation of hematopoietic and stromal cells. Blood 2004; 104: 2202-2203.

29 Rossi DJ, Bryder D, Weissman IL. Hematopoietic stem cell aging: mechanism and consequence. Exp Gerontol. 2007; 42: 385-390.

30 Moody JL, Xu L, Helgason CD, Jirik FR. Anemia, thrombocytopenia, leukocytosis, extramedullary hematopoiesis, and impaired progenitor function in Pten +1 SHIP-/- mice: a novel model of myelodysplasia. Blood 2004; 103: 4503-4510.

31 Louz D, van den Broek M, Verbakel S, Vankan Y, van Lom K, Joosten M et al. Erythroid defects and increased retrovirally-induced tumor formation in Evi1 transgenic mice. Leukemia 2000; 14: 1876-1884.
32 Bench AJ, Aldred MA, Humphray SJ, Champion KM, Gilbert JG, Asimakopoulos FA et al. A detailed physical and transcriptional map of the region of chromosome 20 that is deleted in myeloproliferative disorders and refinement of the common deleted region. Genomics 1998; 49: 351-362.

33 Heim S, Mitelman F. Cytogenetic analysis in the diagnosis of acute leukemia. Cancer 1992; 70: 1701-1709.

34 Qin J, Van Buren D, Huang HS, Zhong L, Mostoslavsky R, Akbarian S et al. Chromatin protein L3MBTL1 is dispensable for development and tumor suppression in mice. J Biol Chem 2010; 285: 27767-27775.

35 Vasikova A, Budinska E, Belickova M, Cermak J, Bruchova H. Differential gene expression of bone marrow CD34 + cells in early and advanced myelodysplastic syndrome. Neoplasma 2009; 56: 335-342.

36 Tefferi A. Pathogenesis of myelofibrosis with myeloid metaplasia. J Clin Oncol 2005; 23: 8520-8530.

37 Down CF, Millour J, Lam EW, Watson RJ. Binding of FoxM1 to G2/M gene promoters is dependent upon B-Myb. Biochim Biophys Acta 2012; 1819 855-862.

38 Santanam U, Zanesi N, Efanov A, Costinean S, Palamarchuk A, Hagan JP et al. Chronic lymphocytic leukemia modeled in mouse by targeted miR-29 expression. Proc Natl Acad Sci USA 2010; 107: 12210-12215.

39 Papaemmanuil E, Cazzola M, Boultwood J, Malcovati L, Vyas $\mathrm{P}$, Bowen D et al. Somatic SF3B1 mutation in myelodysplasia with ring sideroblasts. $N$ Engl J Med 2011; 365: 1384-1395.

40 Visconte V, Makishima H, Jankowska A, Szpurka H, Traina F, Jerez A et al. SF3B1, a splicing factor is frequently mutated in refractory anemia with ring sideroblasts. Leukemia 2012; 26: 542-545.

41 Damm F, Thol F, Kosmider O, Kade S, Loffeld P, Dreyfus F et al. SF3B1 mutations in myelodysplastic syndromes: clinical associations and prognostic implications. Leukemia 2012; 26: 1137-1140.

cc This work is licensed under the Creative Commons AttributionNonCommercial-No Derivative Works 3.0 Unported License. To view copy of this license, visit http://creativecommons.org/licenses/by-nc-nd/3.0/

Supplementary Information accompanies the paper on the Leukemia website (http://www.nature.com/leu) 